\title{
M6A Demethylase FTO Plays a Tumor Suppressor Role in Thyroid Cancer
}

\author{
Run Tian, ${ }^{1, *}$ Shidong Zhang, ${ }^{1, *}$ Daxin Sun, ${ }^{2}$ Chunhua Bei, ${ }^{1}$ Di Li, ${ }^{1}$ Chuanjun Zheng, ${ }^{1}$ Xin Song, \\ Mengshi Chen, ${ }^{3}$ Shengkui Tan, ${ }^{1,4}$ Xiaonian Zhu, and Huixia Zhang ${ }^{1}$
}

The purpose of this study was to investigate the expression and clinical significance of N6-methyladenosine demethylase FTO in thyroid cancer. Bioinformatic analysis showed that FTO expression was downregulated in thyroid cancer tissues and correlated with lymph node metastasis in thyroid cancer patients. We conducted experimental verification by collecting Asian samples. The results of quantitative reverse transcription-PCR showed that the mRNA expression of FTO in the blood of 30 thyroid cancer patients was lower than that of the control population. At the same time, we found that FTO expression was negative in tissues of 16/56 (28.57\%) thyroid cancer cases and 4/40 (10.00\%) nontumor thyroid cases through the immunohistochemical method, indicating a lower FTO expression in thyroid cancer tissues than nontumor thyroid tissues $(p<0.05)$. In addition, the protein expression of FTO was significantly related to the tumor grade and lymph node metastasis in thyroid cancer patients $(p<0.05)$, but not to other clinicopathological features. Multivariate logistic regression analysis showed that FTO expression was an independent risk factor for tumor grade. Survival analysis showed no significant difference in the disease-free survival time of thyroid cancer patients between high expression and low expression groups of FTO. Furthermore, bioinformatic analysis found that promoter DNA methylation and copy number variation might cause downregulated FTO and then affect TP53 pathways in thyroid cancer. We found that FTO expression was downregulated in thyroid cancer tissues and related to the progression of thyroid cancer, suggesting a tumor suppressor role of FTO in thyroid cancer.

Keywords: FTO, m6A modification, thyroid cancer, metastasis, prognosis

\section{Introduction}

TN THE PAST 50 YEARS, the incidence of thyroid cancer has been rising worldwide (Li et al., 2020b). Thyroid cancer is the fifth most common cancer among women in the United States, and in 2020, it was estimated that there were more than 52,890 new cases of men and women (Siegel et al., 2020). From 1990 to 2020, the global incidence of thyroid cancer has continued to rise (Wild et al., 2020). Although the incidence rate has steadily increased, the mortality rate of thyroid cancer has changed little in most countries and regions (Vaccarella et al., 2016; Dal Maso et al., 2018). Compared with high-income countries $(19 \%)$, the relative growth rate of thyroid cancer in low-income countries (33\%) is higher. The reason for this manifestation mainly reflects the increasingly stricter examination of the thyroid by ultra- sound and other diagnostic techniques (Li et al., 2020a). When comparing different regions of the world, the incidence of thyroid cancer seems to be very different due to differences in diagnostic methods, environmental exposure, and individual risk factors (Li et al., 2020b). Because thyroid cancer has the characteristics of strong concealment and slow progress, some patients have tumor metastasis at the initial diagnosis (Ancker et al., 2017). According to reports in the literature, the occurrence of thyroid cancer is related to activation and deregulation of oncogenes, so revealing thyroid cancer-related genes and regulatory mechanisms is of great significance for the treatment of thyroid cancer (Wang et al., 2020).

Studies have shown that N6-methyladenosine (m6A) is the most abundant RNA modification in eukaryotic and human RNA (Desrosiers et al., 1974). Similar to DNA and

\footnotetext{
${ }^{1}$ Department of Epidemiology and Health Statistics, School of Public Health, Guilin Medical University, Guilin, P.R. China.

${ }^{2}$ Department of Thyroid Diseases, The First Affiliated Hospital of Guilin Medical University, Guilin, P.R. China.

${ }^{3}$ Hunan Provincial Key Laboratory of Clinical Epidemiology, Xiangya School of Public Health, Central South University, Changsha, P.R. China.

${ }^{4}$ Department of Epidemiology and Health Statistics, Xiangya School of Public Health, Central South University, Changsha, P.R. China.

*These authors contributed equally to this work.
} 
protein modification, the RNA methylation process is continuous and reversible. In recent years, m6A has attracted more and more scientists' attention due to its great biological significance, prognosis, and therapeutic potential (Cao et al., 2016). The m6A modification can work under the coordinated action of methyltransferase (writers), demethylase (erasers), and reader proteins (Yue et al., 2015). The M6A writer complex comprises METTL3, WTAP, RBM15, METTL14, KIAA1429, and ZC3H13 (Roignant and Soller, 2017; Lan et al., 2019). Demethylase FTO and ALKBH5 constitute m6A erasers (Fitzsimmons and Batista, 2019). M6A readers include YTHDF1, YTHDF2, YTHDC1, YTHDC2, and HNRNPC, which can recognize m6A modifications and thus directly guide alternative splicing, localization, translation, and stability of RNA and other processes (Arguello et al., 2017; Wang et al., 2018).

The obesity and fat-related protein called $\alpha$-ketoglutaratedependent dioxygenase is an enzyme encoded by the human FTO gene (Li et al., 2019a). Animal model studies have shown that FTO deficiency can prevent obesity and cause growth retardation (Fischer et al., 2009; Gao et al., 2010), and overexpression of FTO can lead to increased food intake and obesity (Church et al., 2010). In humans, growth retardation and various malformations or even premature death may be caused by FTO gene mutation or loss of function (Boissel et al., 2009). By demethylating the target mRNA, FTO is involved in the regulation of various tumors ( $\mathrm{Li}$ et al., 2017; Wang et al., 2017). However, the role of FTO in human thyroid cancer is still unknown.

Through clarifying the expression and clinical significance of FTO in thyroid cancer, we aim to explore the biological function of FTO in thyroid cancer and provide new clues for molecular targeted therapy of thyroid cancer.

\section{Materials and Methods}

\section{Blood samples}

Thirty blood samples in the case group were collected from patients with thyroid cancer diagnosed by pathological and clinical methods in the First Affiliated Hospital of Guilin Medical University from July to December 2019. These patients had no thyroid surgery, radiotherapy, or chemotherapy before. At the same time, 30 normal control blood samples were collected from the Department of Physical Examination in Guilin People's Hospital. The control group was matched with the case group according to age and gender and had no thyroid diseases. All the samples were from Asians. This study was based on the 1975 Declaration of Helsinki and was approved by the Ethics Committee of Guilin Medical University (GLMC2014003).

\section{Bioinformatic analysis}

Data of FTO expression in thyroid cancer tissues were obtained from the National Center for Biotechnology Information Gene Expression Omnibus (NCBI GEO) and The Cancer Genome Atlas (TCGA) databases through the GDC platform according to the previous method (Guo et al., 2020). High and low expression groups of FTO were set by the median of the FTO expression value. We used the Kaplan-Meier (KM) plotter for survival analysis. KM analysis was used for relapse-free survival time from TCGA's thyroid cancer patients. FTO DNA methylation and copy number alterations (CNA) in TCGA-thyroid carcinoma (THCA) were studied using cBioPortal for Cancer Genomics. We used the Whistle-Epitranscriptome online tool to predict the m6A methylation site of the TP53 gene (Chen et al., 2019).

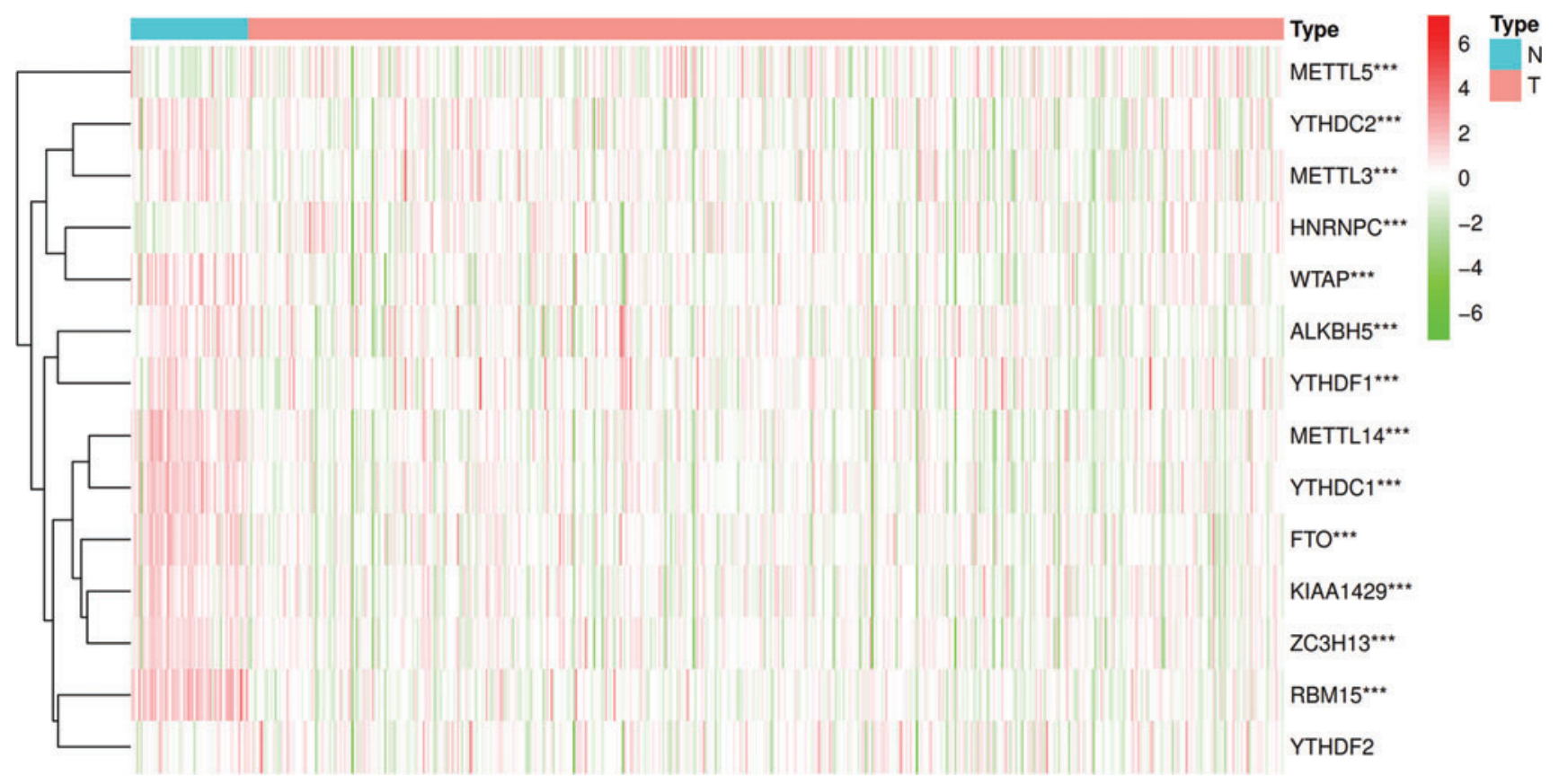

FIG. 1. Expression of $\mathrm{N}$ 6-methyladenosine RNA methylation regulators in thyroid cancer in TCGA database. The Wilcox test was used to determine different gene expression levels between the tumor group and normal group; $* * * p<0.001$. TCGA, The Cancer Genome Atlas. Color images are available online. 
Quantitative reverse transcription-polymerase chain reaction

RNA was isolated from blood samples using the QIAamp RNA Blood Mini Kit (QIAGEN, Germantown, MD) and subsequently used for cDNA synthesis with the FastQuant RT Kit (Tiangen, Beijing, China), according to the manufacturers' protocols. Then, quantitative reverse transcription (qRT)PCR was performed using the StepOne/StepOnePlus ${ }^{\mathrm{TM}}$ Real-Time PCR System with SYBR Green PCR Master mix (Thermo Fisher Scientific, Waltham, MA). The primer sequence for FTO was the following: forward, 5'-GGGTTCAT CCTACAACGGACA-3', and reverse, 5'-AGGTTCCTCTTT CAGGTATGGC- $3^{\prime}$. The GAPDH primers used were the following: forward, 5'-GACCACTTTGTCAAGCTCATTTC-3',

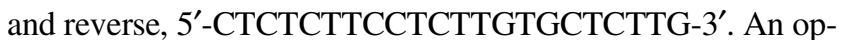
timal reaction was obtained with the following thermocycling conditions: initial denaturation at $95^{\circ} \mathrm{C}$ for $10 \mathrm{~min}$; 45 cycles of denaturation at $95^{\circ} \mathrm{C}$ for $15 \mathrm{~s}$, annealing at $60^{\circ} \mathrm{C}$ for $1 \mathrm{~min}$, and elongation at $72^{\circ} \mathrm{C}$ for $1 \mathrm{~min}$; and a final extension at $72^{\circ} \mathrm{C}$ for $10 \mathrm{~min}$.

\section{Immunohistochemical detection}

The immunohistochemical (IHC) method was performed after tissue specimens were integrated into a tissue chip by the Fanpu company (Guilin, China). After IHC standard steps for baking, dewaxing, and antigen repair, peroxidase blocking, and goat serum incubation, the tissue chip was
FIG. 2. Expression of FTO in thyroid cancer in the online database. (A-D) Data from the GEO dataset. (E, F) Data from TCGA dataset. The $t$-test or paired $t$-test was used to determine different gene expression levels between the tumor group and normal group; $* * p<0.01$ and $* * * p<0.001$. GEO, Gene Expression Omnibus. Color images are available online.
A

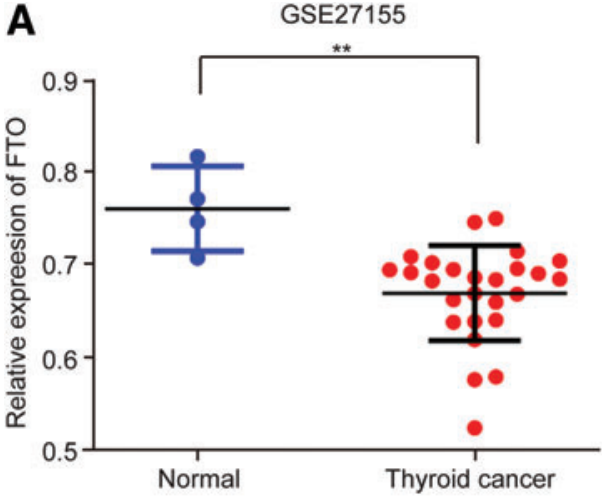

C

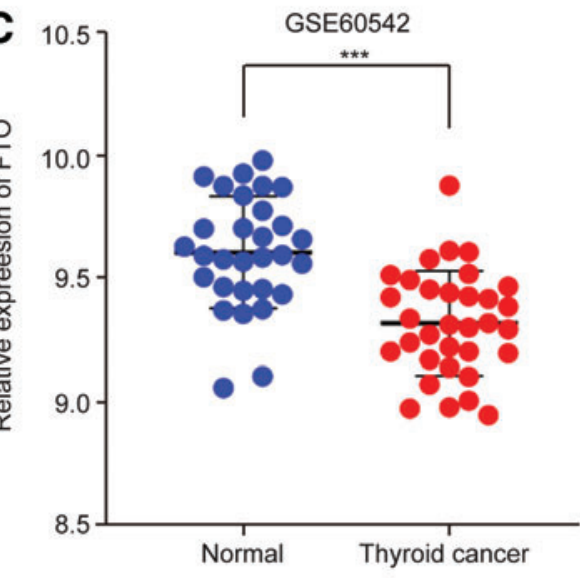

E

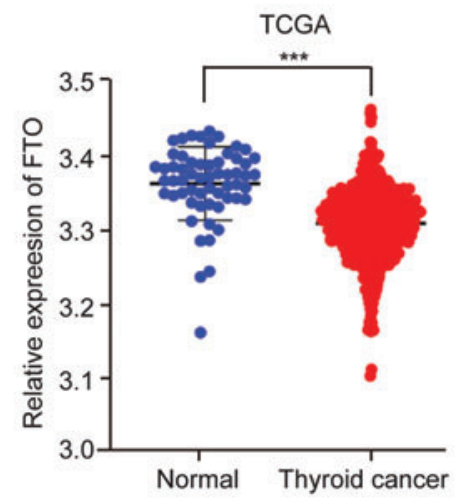

B
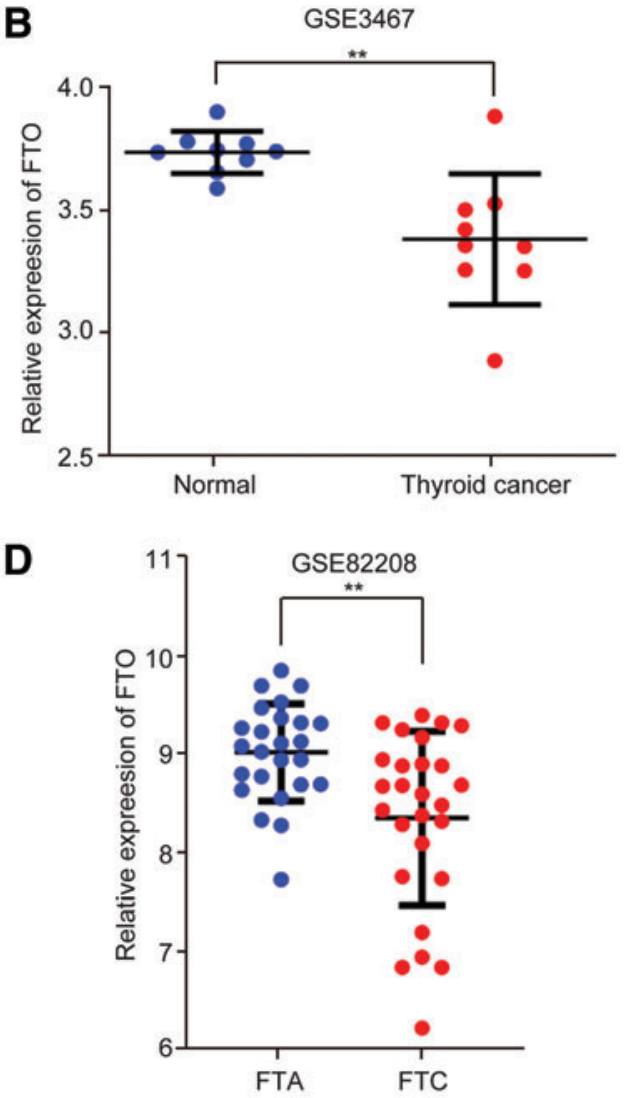

F

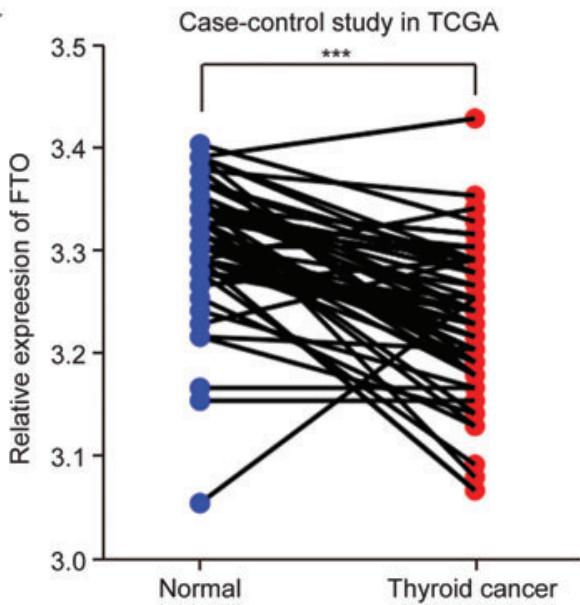


incubated with anti-rabbit polyclonal antibody against FTO (Atlas Antibodies, Bromma, Sweden) overnight at $4^{\circ} \mathrm{C}$. Then, the anti-rabbit secondary antibody was added and incubated at $37^{\circ} \mathrm{C}$ for $30 \mathrm{~min}$. Finally, the diaminobenzidine solution was added to develop color and hematoxylin was added to restaining. The IHC results were determined under a microscope by two professional pathologists. The average staining intensity and percentage of positively stained cells in five visual fields were recorded and counted. The scores for percentage of positively stained cells were 0 for none, 1
A

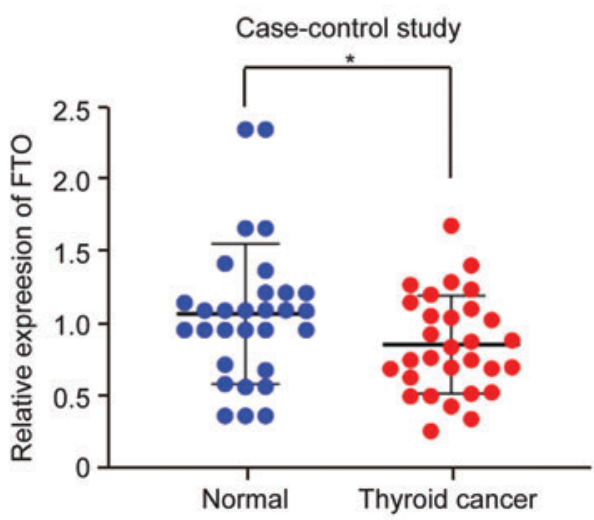

B

FTO (+)

FTO (-)

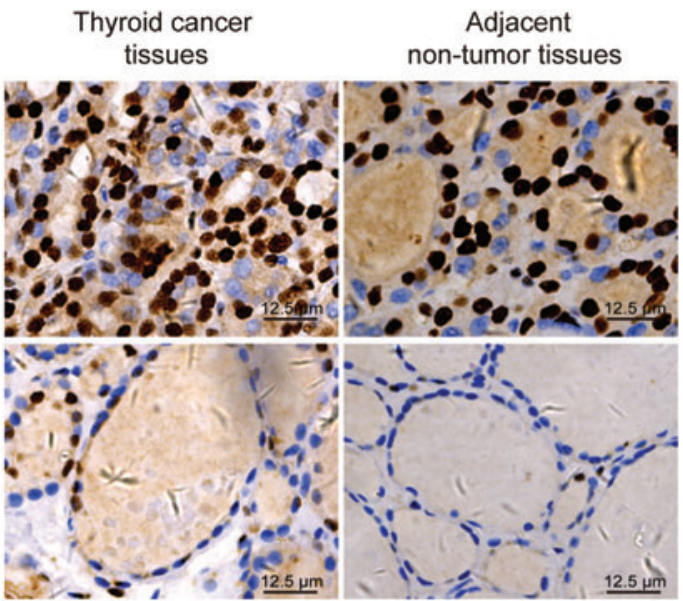

C

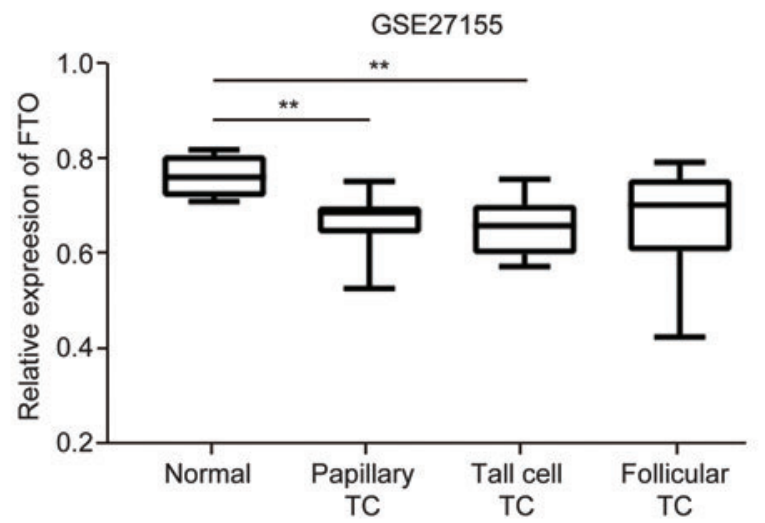

D

TCGA

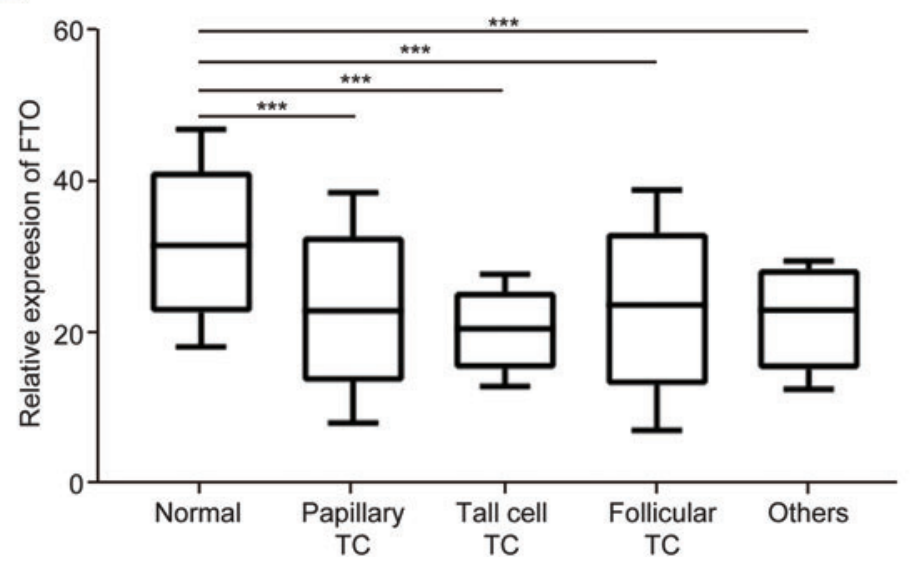

FIG. 3. Experimental verification of FTO expression in patients with thyroid cancer. (A) The expression of FTO in the blood of thyroid cancer patients and nonthyroid cancer patients matched by age and sex was analyzed by qRT-PCR. The paired $t$-test was used to determine different gene expression levels between the tumor group and normal group; $* p<0.05$. (B) Representative FTO protein expression was detected in thyroid cancer tissues and nontumor thyroid tissues by IHC analysis at $400 \times$ magnification. (C) In the GEO database, comparison of the expression of FTO after cancer classification with the normal control group; $* * p<0.01$. (D) In TCGA database, comparison of the expression of FTO after cancer classification with the normal control group; *** $p<0.001$. IHC, immunohistochemical; qRT, quantitative reverse transcription. Color images are available online. 
for $\leq 10 \%, 2$ for $11-50 \%, 3$ for $51-75 \%$, and 4 for $>75 \%$, respectively. The scores for staining strength were 0 for no staining, 1 for light brown, 2 for medium brown, and 3 for dark brown. Finally, the scores for staining strength and percentage of positively stained cells were multiplied to obtain the total expression score. An expression score $\leq 4$ was defined as negative expression and $>4$ was defined as positive expression.

\section{Gene set enrichment analysis}

Gene set enrichment analysis (GSEA) first generated an ordered list of all genes based on their correlation with FTO expression and then we conducted GSEA to clarify the significant differences observed between the high and low expression groups of FTO. The genome was aligned 1000 times per analysis. The expression level of FTO was used as a phenotypic marker. Nominal $p$-value and normalized enrichment score (NES) were used to classify the enrichment pathways in each phenotype.

\section{Statistical analysis}

All data were analyzed using SPSS 24.0. The expression of FTO between thyroid cancer and paired nontumor thyroid tissues was compared by the chi-square test. The relationship between the expression level of FTO and clinicopathological features of thyroid cancer patients was analyzed by the chi-square test and multivariate logistic regression analysis. The KM method was used for survival analysis. $p<0.05$ was considered as statistically significant.

\section{Results}

Expression of $m 6 A$-associated factors in thyroid cancer

According to literature reports, genes of the m6A family play important biological roles in a variety of cancers, but there are few relevant studies in thyroid cancer (Wang et al., 2020; Zhao et al., 2020). To screen out the deregulated m6A-associated factors, we used TCGA database to analyze data of the expression of m6A family transcriptome in thyroid cancer. We chose 14 identified m6A-associated factors in the analysis, METTL3, METTL14, METTL5, ALKBH5, FTO, YTHDC1, YTHDC2, YTHDF1, YTHDF2, HNRNPC, WTAP, KIAA1429, ZC3H13, and RBM15. As shown in Figure 1, except the m6A-binding protein, YTHDF2, all other $13 \mathrm{~m} 6 \mathrm{~A}$-associated factors were differentially expressed in thyroid cancer tissues $(p<0.01)$. This result is consistent with previous research (Xu et al., 2020). Among these $13 \mathrm{~m} 6 \mathrm{~A}$-associated factors, the expression levels of binding proteins, HNRNPC and methyltransferase METTL5, in thyroid cancer tissues were higher than those in adjacent nontumor tissues. Compared with the adjacent nontumor tissues, FTO and the remaining 10 genes of the m6A family were significantly downregulated in thyroid cancer tissues.

\section{Bioinformatic analysis of FTO expression in thyroid cancer}

To further verify the expression of FTO in thyroid cancer, we next conducted bioinformatic analysis to measure the expression of FTO in thyroid cancer patients using TCGA and GEO databases. Compared with the normal thyroid cancer tissues, the expression of FTO was significantly lower in thyroid cancer tissues in GSE27155, GSE3467, GSE60542, and TCGA databases $(p<0.01$, Fig. $2 \mathrm{~A}-\mathrm{C}$ and E). In addition, in GSE82208, FTO expression in follicular thyroid carcinoma was lower than that in follicular thyroid adenoma $(p<0.01$, Fig. 2D). Especially in TCGA database, we further paired the samples to perform a paired $t$-test. As shown in Figure 2F, the expression of FTO in thyroid cancer tissues was still lower than that in adjacent nontumor tissues $(p<0.01)$. These online analyses suggest that FTO has a downregulated expression in thyroid cancer tissues.

\section{Expression of FTO in patients with thyroid cancer}

We collected blood and tissue samples from thyroid cancer patients to further detect the expression of FTO in thyroid cancer and to verify the results of online databases. As shown in Figure 3A, the mRNA expression of FTO in the blood of thyroid cancer patients was lower than that of the matched normal control population $(p<0.05)$. The IHC method was used to detect the expression of FTO protein in thyroid cancer tissues. Results showed that the FTO protein was mainly located in the nucleus (Fig. 3B). Moreover, $28.57 \%(16 / 56)$ of thyroid cancer tissues had negative FTO expression and $10.00 \%$ (4/40) of nontumor thyroid tissues had negative FTO expression. Statistical analysis showed that the negative expression of FTO in thyroid cancer tissues was higher than that in nontumor thyroid tissues $(p<0.05$, Table 1). These results further indicate that FTO is downregulated in thyroid cancer tissues.

We further classified thyroid cancer and found that in TCGA database, the expression of FTO in each type of cancer tissue was lower than that of normal thyroid tissue $(p<0.001$, Fig. 3D). Similarly, in GSE27155, FTO expression in thyroid gland papillary carcinoma and tall cell variant thyroid gland papillary carcinoma was also lower than that in normal thyroid tissues $(p<0.01$, Fig. 3C).

\section{The relationship between FTO expression and clinicopathological features of thyroid cancer patients}

We also analyzed the relationship between FTO expression and clinical characteristics of thyroid cancer patients through online databases and IHC results. From TCGA database, it was found that the expression of FTO was related to lymph

TABle 1. FTO Expression IN Thyroid CANCER Tissues AND ADJACENT NONTUMOR TISSUES By IMmunohistochemicAl Detection

\begin{tabular}{lcccc}
\hline $\begin{array}{l}\text { FTO } \\
\text { expression }\end{array}$ & $\begin{array}{c}\text { Adjacent } \\
\text { nontumor } \\
\text { tissues }\end{array}$ & $\begin{array}{c}\text { Thyroid } \\
\text { cancer } \\
\text { tissues }\end{array}$ & $\chi^{2}$ & $\mathrm{p}$ \\
\hline Negative & 4 & 16 & 4.879 & $\mathbf{0 . 0 2 7}$ \\
Positive & 36 & 40 & & \\
Total & 40 & 56 & & \\
\hline
\end{tabular}

Bold values indicate significance. 

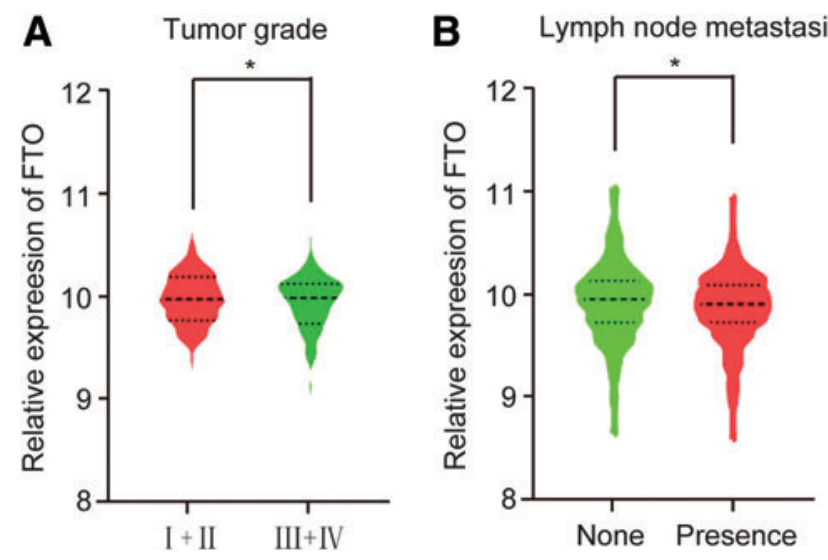

FIG. 4. The relationship between FTO expression and clinicopathological features of thyroid cancer patients analyzed from TCGA database. (A) The relationship between FTO expression and tumor grade of thyroid cancer patients. (B) The relationship between FTO expression and lymph node metastasis in patients with thyroid cancer; $* p<0.05$ is based on the $t$-test. Color images are available online.

node metastasis and tumor grade in patients with thyroid cancer ( $p<0.05$, Fig. 4A, B). According to IHC results, 56 patients with thyroid cancer were divided into FTO-positive and -negative expression groups. As shown in Table 2, the expression of FTO was significantly correlated with age, lymph node metastasis, and tumor grade $(p<0.05)$, but not with other clinicopathological features (such as gender and tumor diameter), in patients with thyroid cancer. Multiple logistic regression analysis further showed that the expression of FTO was related to the tumor grade of thyroid cancer and its odds ratio was $0.38(p<0.01$, Table 3$)$. These results indicate that FTO expression is associated with lymph node metastasis and tumor grade in patients with thyroid cancer.

\section{Table 2. Association Between the Expression of FTO and Clinicopathological Features of Thyroid Cancer Patients Based ON IMMUNOHISTOCHEMICAL RESULTS}

\begin{tabular}{|c|c|c|c|c|c|}
\hline \multirow[b]{2}{*}{ Variables } & \multirow[b]{2}{*}{ Total } & \multicolumn{2}{|c|}{ FTO staining } & \multirow[b]{2}{*}{$\chi^{2}$} & \multirow[b]{2}{*}{$\mathrm{p}$} \\
\hline & & Negative & Positive & & \\
\hline \multicolumn{6}{|l|}{ Gender } \\
\hline Female & 30 & 6 & 24 & 2.326 & 0.127 \\
\hline Male & 26 & 10 & 16 & & \\
\hline \multicolumn{6}{|l|}{ Age, years } \\
\hline$\leq 50$ & 34 & 4 & 30 & 11.979 & 0.001 \\
\hline$>50$ & 22 & 12 & 10 & & \\
\hline \multicolumn{6}{|c|}{ Tumor diameter (cm) } \\
\hline$\leq 4$ & 44 & 10 & 34 & 3.436 & 0.064 \\
\hline$>4$ & 12 & 6 & 6 & & \\
\hline \multicolumn{6}{|c|}{ Tumor grade } \\
\hline $\mathrm{I}+\mathrm{II}$ & 48 & 8 & 40 & 23.333 & $<0.001$ \\
\hline III+IV & 8 & 8 & 0 & & \\
\hline \multicolumn{6}{|c|}{ Lymphatic node metastasis } \\
\hline None & 44 & 9 & 35 & 6.629 & 0.010 \\
\hline Presence & 12 & 7 & 5 & & \\
\hline
\end{tabular}

Bold values indicate significance.
Table 3. Multivariable Logistic Regression ANALYSIS OF FTO EXPRESSION IN THYROID CANCER Patients

\begin{tabular}{|c|c|c|c|c|c|c|}
\hline \multirow[b]{2}{*}{ Variables } & \multirow[b]{2}{*}{ B } & \multirow[b]{2}{*}{ Wald } & \multirow[b]{2}{*}{$\mathrm{p}$} & \multirow[b]{2}{*}{$O R^{\mathrm{a}}$} & \multicolumn{2}{|c|}{$95 \%$ CI for $O R$} \\
\hline & & & & & Lower & Upper \\
\hline FTO & -0.967 & 7.480 & 0.006 & 0.380 & 0.190 & 0.760 \\
\hline
\end{tabular}

\section{The relationship between FTO expression and prognosis of thyroid cancer patients}

Because the prognosis of patients with thyroid cancer is better, we used the KM method to analyze the relationship between FTO expression and the prognosis of thyroid cancer patients online. As shown in Figure 5, according to TCGA database, there was no significant difference in tumor-free survival time between high and low expression groups of FTO after tumor surgery.

\section{Possible mechanism of FTO expression in thyroid cancer}

We also explored the potential mechanisms of FTO downregulation in thyroid cancer from the perspective of genetic and epigenetic alterations. Among thyroid cancer patients with complete mRNA, CNA, and methylation data $(n=497)$ in TCGA-THCA dataset, two patients had FTO shallow deletion and 13 patients had FTO copy gain (lowlevel amplification). FTO copy gain was significantly related to FTO mRNA overexpression $(p<0.05$, Fig. 6B). Besides, we evaluated the association between FTO expression and its DNA methylation. Linear regression analysis showed a significant correlation between FTO mRNA expression and

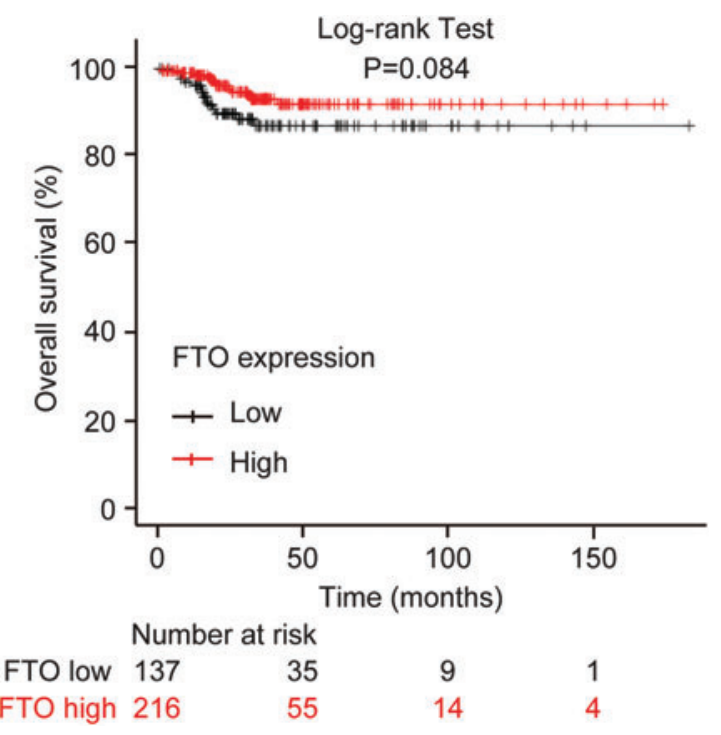

FIG. 5. The relationship between FTO expression and prognosis of thyroid cancer patients from TCGA database. Color images are available online. 
A

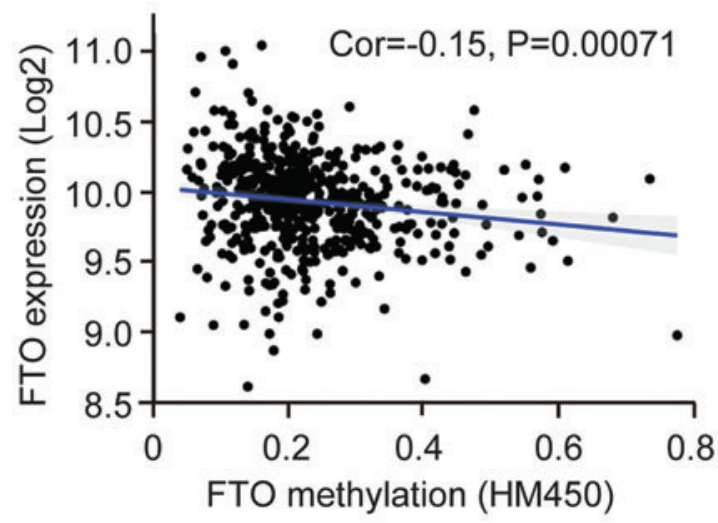

C

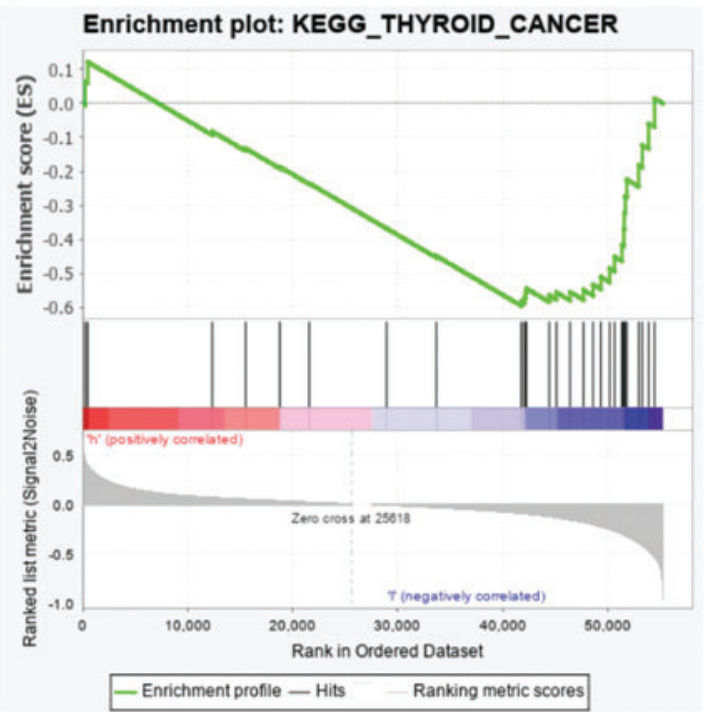

B

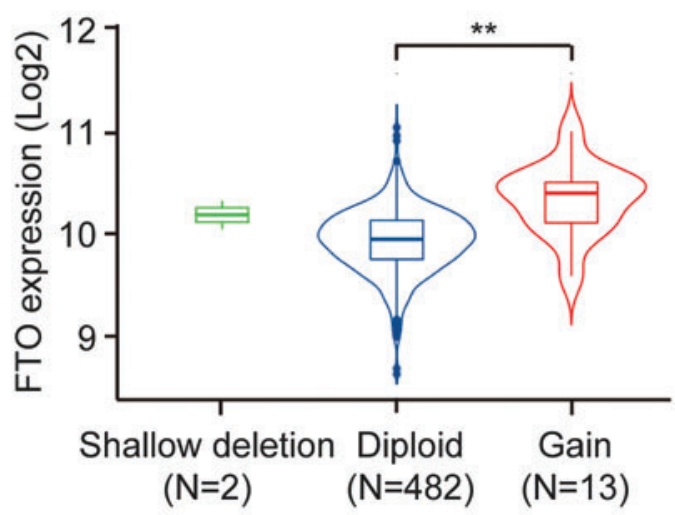

D

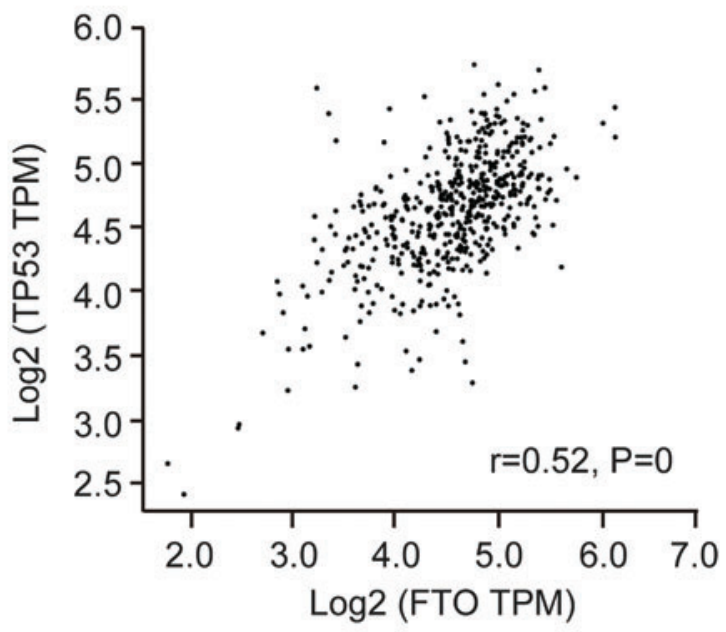

FIG. 6. Possible regulatory mechanism of FTO expression in thyroid cancer. (A) Correlation analysis between FTO mRNA expression and FTO DNA methylation. (B) Comparison of FTO mRNA expression in different CNA groups. (C) GSEA using TCGA-THCA dataset. FTO silence was significantly correlated with thyroid cancer. (D) Correlation between TP53 and FTO in transcriptome data after TPM correction. $* * P<0.01$. CNA, copy number alteration; GSEA, gene set enrichment analysis; THCA, thyroid carcinoma; TPM, transcripts per million. Color images are available online.

the total DNA methylation level (Pearson's $r=-0.15$, $p<0.01$, Fig. 6A). Moreover, we established a link between the methylation site and expression of FTO. The results showed that the methylation sites, cg12495954, cg01485549, $\operatorname{cg} 06392242$, and cg27021131, would affect the expression of FTO (Supplementary Fig. S1).

To identify signaling pathways that FTO may participate in for thyroid cancer, we conducted GSEA between low and high expression datasets of FTO. GSEA revealed significant differences in enrichment of KEGG (FDR $q<0.05$, NOM $p<0.01)$. We selected the most significant enriched signaling pathways based on their NES (Fig. 6C and Table 4). After the GSEA, we found that the expression of FTO might affect the KEGG THYROID CANCER pathway. We tried a correlation between FTO and the key gene, TP53, in the KEGG THYROID CANCER pathway in patients with thyroid cancer and found that FTO and TP53 were positively correlated. Online prediction of the m6A methylation site of TP53 through the Whistle website found that there were several m6A methylation sites on TP53 (Supplementary Table S1). Therefore, we speculate that FTO may affect the metastasis of thyroid cancer by affecting the KEGG THYROID CANCER signaling pathway through targeting m6A methylation of TP53 (Fig. 6D).

\section{Discussion}

In all RNA modification processes, RNA methylation accounts for more than $60 \%$, and $\mathrm{m} 6 \mathrm{~A}$ is recognized as one of the most common types of eukaryotic mRNA methylation modifications (Li et al., 2019b). At present, three enzymes have been identified to participate in the m6A methylation process, including demethylase, methylase, and methylation recognition enzyme (Bartosovic et al., 2017). Among them, m6A demethylase comprises FTO and ALKBH5 (Zhao et al., 2020). As the first RNA demethylase identified, FTO is reported to regulate many cellular processes, such as adipogenesis, mRNA splicing, and cancer development (Iles 
Table 4. Gene Sets Enriched in Phenotype with High FTO Expression

\begin{tabular}{|c|c|c|c|c|}
\hline Name & Size & $N E S$ & $N O M$ p-val & $F D R$ q-va \\
\hline KEGG_WNT_SIGNALING_PATHWAY & 150 & -2.16318 & 0 & 0.001723 \\
\hline KEGG_TGF_BETA_SIGNALING_PATHWAY & 85 & -2.1385 & 0 & 0.003128 \\
\hline KEGG_PROSTATE_CANCER & 89 & -2.10176 & 0 & 0.005823 \\
\hline KEGG_INSULIN_SIGNALING_PATHWAY & 137 & -2.08895 & 0 & 0.004367 \\
\hline KEGG_COLORECTAL_CANCER & 62 & -2.08428 & 0 & 0.003494 \\
\hline KEGG_ADHERENS_JUNCTION & 73 & -2.08084 & 0 & 0.002911 \\
\hline KEGG_ERBB_SIGNALING_PATHWAY & 87 & -2.07179 & 0 & 0.002496 \\
\hline KEGG_INOSITOL_PHOSPHATE_METABOLISM & 54 & -2.07178 & 0 & 0.002184 \\
\hline KEGG_LYSINE_DEGRADATION & 44 & -2.07048 & 0 & 0.001941 \\
\hline KEGG_VASOPRESSIN_REGULATED_WATER_REABSORPTION & 44 & -2.06472 & 0 & 0.002094 \\
\hline KEGG_MTOR_SIGNALING_PATHWĀY & 52 & -2.05036 & 0 & 0.002697 \\
\hline KEGG_ADIPOCYTOKINE_SIGNALING_PATHWAY & 67 & -2.00841 & 0 & 0.004424 \\
\hline KEGG_PHOSPHATIDYLINOSITOL_SIGNALING_SYSTEM & 76 & -1.98014 & 0.002012 & 0.006921 \\
\hline KEGG_LONG_TERM_POTENTIATION & 70 & -1.96732 & 0 & 0.008028 \\
\hline KEGG_ENDOMETRIAL_CANCER & 52 & -1.95239 & 0.00207 & 0.009158 \\
\hline KEGG_DORSO_VENTRĀL_AXIS_FORMATION & 24 & -1.94849 & & 0.009134 \\
\hline KEGG_UBIQUITIN_MEDIATED_PROTEOLYSIS & 134 & -1.94066 & 0.004032 & 0.009273 \\
\hline KEGG_NEUROTROPHIN_SIGNALING_PATHWAY & 126 & -1.93574 & 0.006048 & 0.009454 \\
\hline KEGG_PATHWAYS_IN_CANCER & 325 & -1.92563 & 0 & 0.009923 \\
\hline KEGG_VASCULAR_SMOOTH_MUSCLE_CONTRACTION & 115 & -1.92032 & 0.001992 & 0.010206 \\
\hline KEGG_MELANOGENESIS & 101 & -1.91057 & 0 & 0.010899 \\
\hline KEGG_RENAL_CELL_CARCINOMA & 70 & -1.91011 & 0.002041 & 0.010493 \\
\hline KEGG_PANCREATIC_CANCER & 70 & -1.90877 & 0.004 & 0.010174 \\
\hline İ_SPHINGOLIPID__METABOLISM & 39 & -1.90253 & 0 & 0.010619 \\
\hline KEGG_GNRH_SIGNALING_PATHWAY & 101 & -1.89992 & 0 & 0.010654 \\
\hline KEGG_SI & 84 & -1.89 & 0.00396 & 0.010328 \\
\hline KEGG_MAPK_SIGNALING_PATHWAY & 267 & -1.89907 & 0.0 & 0.009997 \\
\hline IHOG_SIGNALING_PATHWAY & 56 & -1.89773 & & 0.00973 \\
\hline KEGG_ENDOCYTOSIS & 181 & -1.89215 & 0.00 & 0.010083 \\
\hline KEGG_F & 199 & & 0.00 & 0.010008 \\
\hline $\mathrm{KEGG}_{\mathrm{C}} \mathrm{H}$ & 44 & -1.87848 & 0 & 0.011053 \\
\hline F_AUTOPHAGY & 35 & -1.86 & 0.001 & 0.013473 \\
\hline OSȲNTHESIS & 46 & -1.86282 & & 0.013245 \\
\hline KEGG_GAP_JUN & 90 & -1.85 & & 0.014413 \\
\hline KEGG_O_GLYC & 30 & -1.84649 & 0.00 & 0.014426 \\
\hline KEGG_PROGESTERONE_MEDIATED_OOCYTE_MATURATION & 85 & -1.83695 & 0.006 & 0.014921 \\
\hline KEGG_TYPE_II_DIABETES_MELLITUSS & 47 & -1.83182 & 0.001 & 0.015452 \\
\hline KEGG_AXON__GUIDANC & 129 & -1.81561 & 0.010661 & 0.017908 \\
\hline KEGG_OOCYT̄E_MEIOS & 112 & -1.81074 & 0.008282 & 0.01847 \\
\hline KEGG_THYROID_CAN & 29 & -1.80713 & 0.0039 & 0.018437 \\
\hline KEGG_CHRONIC_MYELOID_LEUKEMIA & 73 & -1.79938 & & 0.019905 \\
\hline $\begin{array}{l}\text { KEGG_GLYCOSYLPHOSPHATIDYLINOSITOL_GPI_ANCHOR_ } \\
\text { BIOSYNTHESIS }\end{array}$ & 25 & -1.7958 & 0.005703 & 0.02003 \\
\hline KEGG_REGULATION_OF_ACTIN_CYTOSKELETON & 213 & & & \\
\hline KEGG_NON_SMALL_CELL_LUNG__CANCER & 54 & -1.79158 & 0.008197 & 0.019998 \\
\hline
\end{tabular}

Gene sets with NOM $p$-val $<0.01$ and FDR $q$-val $<0.5$ are considered as significant.

FDR, false discovery rate; NES, normalized enrichment score; NOM, nominal.

et al., 2013; Ben-Haim et al., 2015; Li et al., 2017). At present, we understand that m6A methylation can affect the occurrence and development of tumors (Yue et al., 2015). After searching relevant literature, we found that FTO has some biological functions in lung cancer, colorectal cancer, breast cancer, and gastric cancer (Tan et al., 2015; Li et al., 2019a, 2019c; Liu et al., 2019). However, thyroid cancerrelated research has not been found. In addition, the pathogenesis of thyroid cancer is complex. From the perspective of molecular pathology, its occurrence and development are similar to other malignant tumors and it is related to the abnormal expression of oncogenes and tumor suppressor genes at different stages (Ramirez-Moya et al., 2018).
In this study, we first analyzed TCGA and GEO databases and found that FTO mRNA levels in thyroid cancer tissues were relatively lower than in normal tissues. Because there were few Asian patients in TCGA and GEO databases, we collected Asian samples from the hospital and conducted qRT-PCR/IHC experiments. Results showed that FTO mRNA levels in the blood of patients with thyroid cancer were downregulated. Subsequent IHC results showed that the FTO protein was expressed in the nucleus. Compared with nontumor tissues, the protein expression of FTO in thyroid cancer tissues was also reduced. In addition, by analyzing the clinical information of thyroid cancer patients in TCGA database, we concluded that the expression of 
FTO had a negative relationship with the tumor grade. Moreover, the expression of FTO in patients with lymph node metastasis was relatively lower than that in patients without lymph node metastasis. After multivariate logistic regression analysis, we found that the expression of FTO was an independent risk factor for tumor grade of thyroid cancer. Among patients collected for IHC detection, patients with lymph node metastasis also had a lower positive rate of FTO protein than patients without lymph node metastasis. Consistent with the bioinformatic results, as the tumor grade increased, the expression of FTO protein also decreased relatively.

From results of the KM survival analysis using data of TCGA database, we can see that disease-free survival in the low expression group of FTO was generally lower compared with the high expression group of FTO, although the difference between the two groups was not significant. We assume this phenomenon might be due to the reasons that the general prognosis of thyroid cancer is good and it is more difficult to track disease-free survival, which causes more data loss.

As we expected, CNV and DNA methylation both affect FTO expression. Although this association is relatively weak, it also provides a certain direction for subsequent research. Based on the above results, we speculated that the expression of FTO might decrease with progression and metastasis of thyroid cancer. Therefore, through GSEA, we tried to find the possible regulatory mechanism of FTO in thyroid cancer. From results of the GSEA, we can see that the expression of FTO may be involved in regulation of many cancer-related pathways. We screened the most relevant thyroid cancer pathway, KEGG THYROID CANCER, and found that this pathway was related to metastasis of thyroid cancer. Through correlation analysis of the corrected transcriptome data, we concluded that the key protein, TP53, in this pathway was positively correlated with FTO, which further proved our speculation. However, whether and how FTO participates in regulation of these pathways need more in-depth research.

\section{Conclusion}

In conclusion, we found downregulated expression of FTO in thyroid cancer and the expression of FTO may be a potential prognostic molecular marker for thyroid cancer. Further experimental verification should be conducted to prove the biological impact of FTO.

\section{Disclosure Statement}

No competing financial interests exist.

\section{Funding Information}

This work was supported by the National Natural Science Foundation of China (81860586, 81860602), Natural Science Foundation of Guangxi Province (2018GXNSFAA281054, 2018GXNSFBA281216), Key Science and Technology Research and Development Program Project of Guangxi (AB17292074), and Hunan Provincial Key Laboratory of Clinical Epidemiology.

\section{Supplementary Material}

Supplementary Figure S1

Supplementary Table S1

\section{References}

Ancker, O.V., Wehland, M., Bauer, J., Infanger, M., and Grimm, D. (2017). The adverse effect of hypertension in the treatment of thyroid cancer with multi-kinase inhibitors. Int J Mol Sci 18, 625.

Arguello, A.E., DeLiberto, A.N., and Kleiner, R.E. (2017). RNA chemical proteomics reveals the N(6)-methyladenosine (m(6)A)-regulated protein-RNA interactome. J Am Chem Soc 139, 17249-17252.

Bartosovic, M., Molares, H.C., Gregorova, P., Hrossova, D., Kudla, G., and Vanacova, S. (2017). N6-methyladenosine demethylase FTO targets pre-mRNAs and regulates alternative splicing and $3^{\prime}$-end processing. Nucleic Acids Res 45, 11356-11370.

Ben-Haim, M.S., Moshitch-Moshkovitz, S., and Rechavi, G. (2015). FTO: linking m6A demethylation to adipogenesis. Cell Res 25, 3-4.

Boissel, S., Reish, O., Proulx, K., Kawagoe-Takaki, H., Sedgwick, B., Yeo, G.S., et al. (2009). Loss-of-function mutation in the dioxygenase-encoding FTO gene causes severe growth retardation and multiple malformations. Am J Hum Genet 85, 106-111.

Cao, G., Li, H.B., Yin, Z., and Flavell, R.A. (2016). Recent advances in dynamic m6A RNA modification. Open Biol 6, 160003.

Chen, K., Wei, Z., Zhang, Q., Wu, X., Rong, R., Lu, Z., et al. (2019). WHISTLE: a high-accuracy map of the human N6methyladenosine (m6A) epitranscriptome predicted using a machine learning approach. Nucleic Acids Res 47, e41.

Church, C., Moir, L., McMurray, F., Girard, C., Banks, G.T., Teboul, L., et al. (2010). Overexpression of Fto leads to increased food intake and results in obesity. Nat Genet 42, 1086-1092.

Dal Maso, L., Panato, C., Franceschi, S., Serraino, D., Buzzoni, C., Busco, S., et al. (2018). The impact of overdiagnosis on thyroid cancer epidemic in Italy, 1998-2012. Eur J Cancer 94, 6-15.

Desrosiers, R., Friderici, K., and Rottman, F. (1974). Identification of methylated nucleosides in messenger RNA from Novikoff hepatoma cells. Proc Natl Acad Sci U S A 71, 3971-3975.

Fischer, J., Koch, L., Emmerling, C., Vierkotten, J., Peters, T., Bruning, J.C., et al. (2009). Inactivation of the Fto gene protects from obesity. Nature 458, 894-898.

Fitzsimmons, C.M., and Batista, P.J. (2019). It's complicated... $\mathrm{m}(6) \mathrm{A}$-dependent regulation of gene expression in cancer. Biochim Biophys Acta Gene Regul Mech 1862, 382-393.

Gao, X., Shin, Y.H., Li, M., Wang, F., Tong, Q., and Zhang, P. (2010). The fat mass and obesity associated gene FTO functions in the brain to regulate postnatal growth in mice. PLoS One 5, e14005.

Guo, X., Zhang, S., Tan, S., Bei, C., Zhang, H., Zhu, X., et al. (2020). Downregulated CMTM2 poses potential clinical significance in hepatocellular carcinoma. DNA Cell Biol 39, 683-689.

Iles, M.M., Law, M.H., Stacey, S.N., Han, J., Fang, S., Pfeiffer, R., et al. (2013). A variant in FTO shows association with melanoma risk not due to BMI. Nat Genet 45, 428-432, $432 \mathrm{e} 421$. 
Lan, Q., Liu, P.Y., Haase, J., Bell, J.L., Huttelmaier, S., and Liu, T. (2019). The critical role of RNA m(6)A methylation in cancer. Cancer Res 79, 1285-1292.

Li, J., Han, Y., Zhang, H., Qian, Z., Jia, W., Gao, Y., et al. (2019a). The m6A demethylase FTO promotes the growth of lung cancer cells by regulating the m6A level of USP7 mRNA. Biochem Biophys Res Commun 512, 479-485.

Li, J., Yang, X., Qi, Z., Sang, Y., Liu, Y., Xu, B., et al. (2019b). The role of mRNA m(6)A methylation in the nervous system. Cell Biosci 9, 66.

Li, M., Brito, J.P., and Vaccarella, S. (2020a). Long-term declines of thyroid cancer mortality: an international ageperiod-cohort analysis. Thyroid 30, 838-846.

Li, M., Maso, L.D., and Vaccarella, S. (2020b). Global trends in thyroid cancer incidence and the impact of overdiagnosis. Lancet Diabetes Endocrinol 8, 468-470.

Li, Y., Zheng, D., Wang, F., Xu, Y., Yu, H., and Zhang, H. (2019c). Expression of demethylase genes, FTO and ALKBH1, is associated with prognosis of gastric cancer. Dig Dis Sci 64, 1503-1513.

Li, Z., Weng, H., Su, R., Weng, X., Zuo, Z., Li, C., et al. (2017). FTO plays an oncogenic role in acute myeloid leukemia as a N(6)-methyladenosine RNA demethylase. Cancer Cell 31, 127-141.

Liu, X., Liu, L., Dong, Z., Li, J., Yu, Y., Chen, X., et al. (2019). Expression patterns and prognostic value of m(6)A-related genes in colorectal cancer. Am J Transl Res 11, 3972-3991.

Ramirez-Moya, J., Wert-Lamas, L., and Santisteban, P. (2018). MicroRNA-146b promotes PI3K/AKT pathway hyperactivation and thyroid cancer progression by targeting PTEN. Oncogene 37, 3369-3383.

Roignant, J.Y., and Soller, M. (2017). m(6)A in mRNA: an ancient mechanism for fine-tuning gene expression. Trends Genet 33, 380-390.

Siegel, R.L., Miller, K.D., and Jemal, A. (2020). Cancer statistics, 2020. CA Cancer J Clin 70, 7-30.

Tan, A., Dang, Y., Chen, G., and Mo, Z. (2015). Overexpression of the fat mass and obesity associated gene (FTO) in breast cancer and its clinical implications. Int J Clin Exp Pathol 8, 13405-13410.

Vaccarella, S., Franceschi, S., Bray, F., Wild, C.P., Plummer, M., and Dal Maso, L. (2016). Worldwide thyroid-cancer epidemic? the increasing impact of overdiagnosis. N Engl J Med 375, 614-617.

Wang, K., Jiang, L., Zhang, Y., and Chen, C. (2020). Progression of thyroid carcinoma is promoted by the m6A methyltransferase METTL3 through regulating m(6)A methylation on TCF1. Onco Targets Ther 13, 1605-1612.

Wang, S., Chai, P., Jia, R., and Jia, R. (2018). Novel insights on m(6)A RNA methylation in tumorigenesis: a double-edged sword. Mol Cancer 17, 101.

Wang, X., Li, Z., Kong, B., Song, C., Cong, J., Hou, J., et al. (2017). Reduced m(6)A mRNA methylation is correlated with the progression of human cervical cancer. Oncotarget $\mathbf{8}$, 98918-98930.

Wild, C.P., Weiderpass, E., and Stewart, B.W., eds. (2020). World Cancer Report: Cancer Research for Cancer Prevention. (International Agency for Research on Cancer, Lyon, France).

Xu, N., Chen, J., He, G., Gao, L., and Zhang, D. (2020). Prognostic values of m6A RNA methylation regulators in differentiated thyroid carcinoma. J Cancer 11, 5187-5197.

Yue, Y., Liu, J., and He, C. (2015). RNA N6-methyladenosine methylation in post-transcriptional gene expression regulation. Genes Dev 29, 1343-1355.

Zhao, W., Qi, X., Liu, L., Ma, S., Liu, J., and Wu, J. (2020). Epigenetic regulation of $\mathrm{m}(6) \mathrm{A}$ modifications in human cancer. Mol Ther Nucleic Acids 19, 405-412.

Address correspondence to: Shengkui Tan, MS

Department of Epidemiology and Health Statistics School of Public Health Guilin Medical University

1 Zhiyuan Road Guilin 541199 P.R. China

E-mail: sktan2008@sina.cn

Xiaonian Zhu, MS Department of Epidemiology and Health Statistics School of Public Health

Guilin Medical University

1 Zhiyuan Road

Guilin 541199

P.R. China

E-mail: zhuxiaonian0403@163.com

Received for publication July 22, 2020; received in revised form September 3, 2020; accepted September 8, 2020. 